\title{
La psoriasis: de la investigación básica y clínica al desarrollo de nuevos tratamientos
}

\author{
Roberto Esquivel-García, ${ }^{1}$ Gabino Estévez-Delgado, ${ }^{1}$ Alain Raimundo Rodríguez-Orozco, ${ }^{2}$ \\ Alejandra Ochoa-Zarzosa ${ }^{3}$ y Martha Estrella García-Pérez ${ }^{1}$ \\ ${ }^{1}$ Facultad de Químico-Farmacobiología; ${ }^{2}$ Facultad de Ciencias Médicas y Biológicas; ${ }^{3}$ Facultad de Medicina Veterinaria y Zootecnia, Centro \\ Multidisciplinario de Estudios en Biotecnología. Universidad Michoacana de San Nicolás de Hidalgo, Michoacán, México
}

\section{Resumen}

La psoriasis es una enfermedad cutánea incurable que afecta a $2.9 \%$ de la población mexicana, por lo que es trascendente analizar el impacto de la medicina traslacional en el desarrollo de medicamentos antipsoriásicos. En esta revisión se discuten conceptos etiopatogénicos de la enfermedad y se analizan artículos publicados entre 2005 y 2017 en torno a medicamentos en desarrollo, además, se presenta un análisis crítico sobre las perspectivas futuras en el desarrollo de nuevos tratamientos. El uso de estrategias bidireccionales de la medicina traslacional ha permitido incrementar significativamente el número de tratamientos antipsoriásicos disponibles. Se encontraron 18 nuevos fármacos en exploración. La caracterización de antígenos responsables de la activación inmunológica, la identificación de biomarcadores predictivos de eficacia farmacológica, el desarrollo de modelos más representativos de la enfermedad, así como la integración de aspectos farmacogenómicos a estrategias de medicina traslacional fueron identificados como elementos relevantes que deben ser incorporados en el desarrollo de nuevas opciones terapéuticas.

PALABRAS CLAVE: Psoriasis. Objetivos farmacológicos. Medicamentos. Medicina traslacional.

\begin{abstract}
Psoriasis is an incurable cutaneous disease that affects $2.9 \%$ of the Mexican population, and it is therefore important for the impact of translational medicine on the development of anti-psoriatic drugs to be analyzed. In this review, current etiopathogenic concepts of the disease are discussed, and articles on drugs under development published between 2005 and 2017 are reviewed; in addition, a critical analysis on future perspectives for the development new treatments is presented. The use of translational medicine bi-directional strategies of has allowed to significantly increase the number of available anti-psoriatic therapies. Eighteen new investigational drugs were found. Characterization of antigens responsible for immune activation, identification of predictive biomarkers with pharmacologic efficacy, and the development of more representative disease models, as well as the integration of pharmacogenomic aspects to translational medicine strategies were identified as relevant aspects that should be incorporated in the development of new therapeutic options.
\end{abstract}

KEY WORDS: Psoriasis. Pharmacological targets. Drugs. Translational medicine.

Fecha de recepción: 13-01-2017

Fecha de aceptación: 27-09-2017

DOI://dx.doi.org/10.24875/GMM.17003182
Gac Med Mex. 2018;154:502-508

Disponible en PubMed www.gacetamedicademexico.com 


\section{Introducción}

La psoriasis es una enfermedad cutánea incurable de naturaleza inflamatoria caracterizada por placas eritematosas. Se estima que afecta de 2 a $3 \%$ de la población mundial; es menos frecuente en las regiones ecuatoriales que en las nórdicas. ${ }^{1}$ En México hay pocos estudios epidemiológicos sobre la enfermedad, por lo que la magnitud real del problema sigue siendo desconocida. En un simposio celebrado en 2009 en Dallas, Texas, se estimó que la prevalencia en México sería de $2.9 \%,{ }^{2}$ lo que equivaldría a más de 3 millones de mexicanos afectados. La incidencia de muerte debida directamente a la psoriasis es baja, pero sus manifestaciones físicas y psicológicas la hacen incapacitante, comparable al cáncer, la diabetes y la depresión. ${ }^{3}$

Los tratamientos antipsoriásicos actuales se dirigen fundamentalmente al manejo de los síntomas e incluyen fototerapia, así como medicamentos tópicos y sistémicos orientados $\mathrm{a}:{ }^{4}$

- Inhibir la hiperproliferación de los queratinocitos (análogos de la vitamina D).

- Normalizar el proceso de diferenciación de los queratinocitos (análogos de la vitamina D, retinoides).

- Disminuir el reclutamiento y activación de los inmunocitos (ciclosporina $A$, medicamentos biotecnológicos).

- Neutralizar las citocinas proinflamatorias (medicamentos biotecnológicos).

Sin embargo, su utilización a largo plazo está limitada por la pérdida de eficacia, la toxicidad y los elevados costos. Como consecuencia, de 31 a $70 \%$ de los pacientes con psoriasis se encuentran altamente insatisfechos. ${ }^{5}$ La industria farmacéutica está cada vez más interesada en el desarrollo de nuevos tratamientos.

En los últimos años ha nacido el término "medicina traslacional", que se refiere a un nuevo paradigma basado en la necesidad de integrar los conocimientos científicos que se generan durante la investigación básica al desarrollo de tratamientos con impacto clínico (bench-to-bedside). Se trata de un concepto bidireccional, donde los nuevos hallazgos derivados de la investigación clínica sirven de base para la generación de hipótesis científicas utilizadas en ciencia fundamental (bedside-to-bench). Bajo este modelo se utilizan saberes derivados de la genómica, proteómica, medicina y farmacología en aras de conocer con mayor profundidad la fisiopatología de las enfermedades, definir los posibles objetivos farmacológicos y obtener moléculas prometedoras, aportando una visión realista orientada a la transferencia de conocimientos entre diferentes áreas del conocimiento, para generar fármacos cada vez más eficaces.

La psoriasis constituye un excelente ejemplo de la importancia de la medicina traslacional para el desarrollo de nuevos tratamientos. De hecho, los primeros tratamientos antipsoriásicos, como la fototerapia o la ciclosporina, se desarrollaron a partir de una aproximación empírica que partió de la evidencia clínica. Esto conllevó a la generación de hipótesis etiopatogénicas elucidadas posteriormente en investigación básica. ${ }^{6}$

En la actualidad, el desarrollo de tratamientos para la psoriasis ha experimentado un cambio de paradigma, promovido por un mayor conocimiento sobre las causas de la enfermedad, lo que se ha evidenciado con la salida al mercado de medicamentos biotecnológicos dirigidos a neutralizar quimiocinas y citocinas específicas.

La presente revisión tiene como objetivo analizar el impacto de la medicina traslacional en el desarrollo de medicamentos antipsoriásicos. La fisiopatología de la psoriasis se examina con base en los conceptos actuales. Además, se explora la literatura científica sobre nuevos medicamentos en desarrollo y sus objetivos farmacológicos para ofrecer una visión actualizada del desarrollo farmacéutico en esta patología. Finalmente, se presenta un análisis crítico sobre las perspectivas futuras en el desarrollo de nuevos tratamientos para esta enfermedad.

\section{Conceptos etiopatogénicos actuales de la psoriasis}

La etiología de la psoriasis es compleja y multifactorial, ya que implica la interacción compleja entre las células constitutivas de la piel y las respuestas innata y adaptativa del sistema inmunológico (Figura 1). ${ }^{7}$ Las primeras hipótesis consideraban que el defecto primario causante de la enfermedad consistía en los queratinocitos. De hecho, hasta hoy día se considera que la hiperproliferación de estas células es una característica distintiva de la patología. La división rápida de los queratinocitos psoriásicos comparativamente a los normales (siete a 10 días versus 28 a 50 días) conlleva aumento del grosor de la epidermis y cambios en la expresión de los marcadores de diferenciación. ${ }^{8}$ La capa granulosa de la piel, donde comienza la 


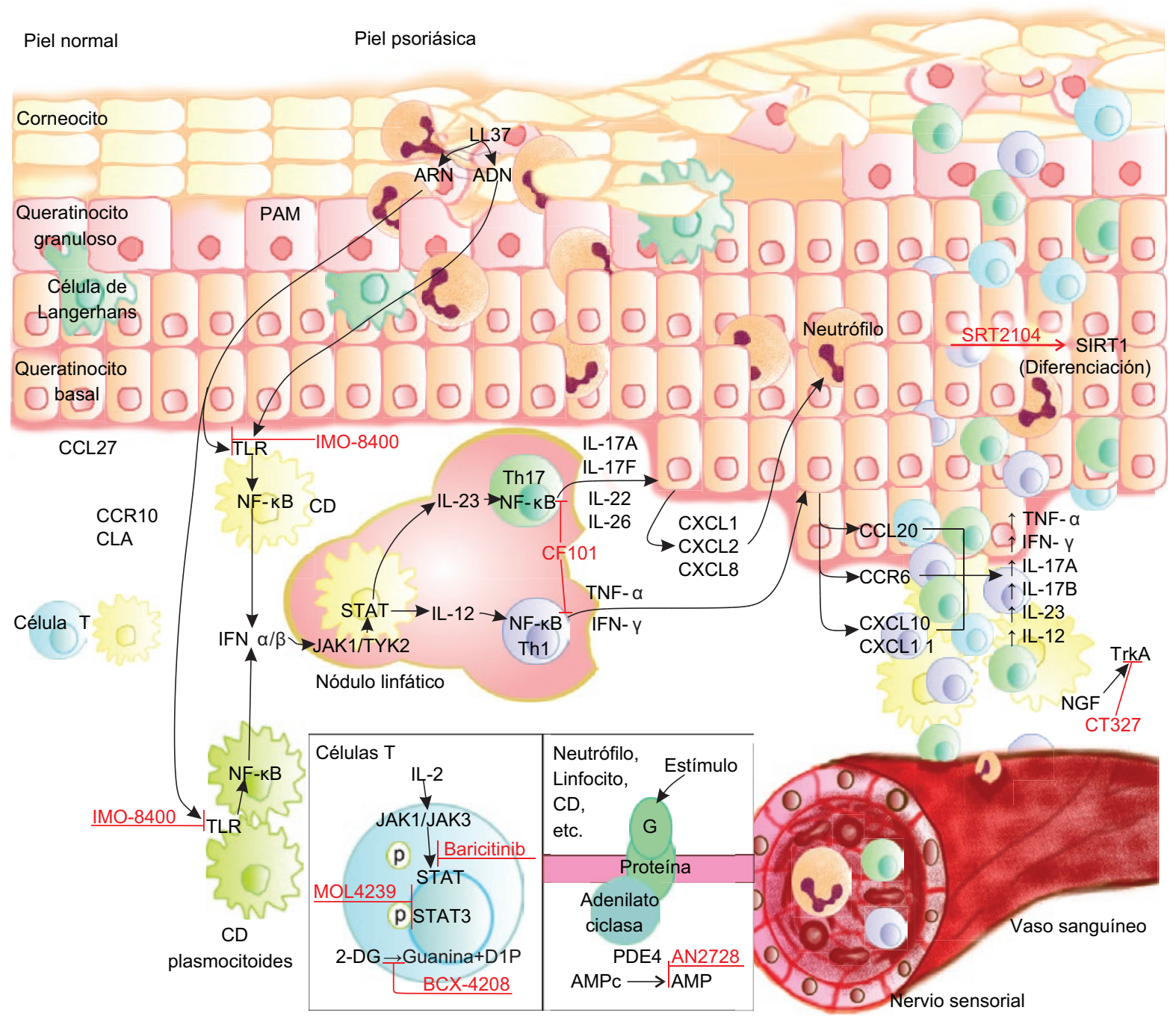

Figura 1. Patogénesis de la psoriasis e implicación de los nuevos fármacos en desarrollo. La psoriasis implica la interacción compleja entre neutrófilos, células dendríticas (CD), linfocitos T (Th1 y Th17) y queratinocitos. La activación de los queratinocitos por una lesión inicial induce la producción de péptidos antimicrobianos (PAM), propiciando que las CD liberen citocinas (IFN- $\alpha$ y $\beta$, IL-12 e IL-23) implicadas en la diferenciación de linfocitos Th1 y Th17, que producen mediadores (IL-17A, IL-17F, IL-22, TNF- $\alpha$ e IFN- $\beta$ ) que estimulan la generación de quimiocinas (CXCL y $C C L$ ) en los queratinocitos, con el consecuente reclutamiento de más inmunocitos en la piel. Algunos de los nuevos fármacos antipsoriásicos en investigación actúan en: a) el bloqueo de la vía de señalización JAK/STAT (baricitinib, MOL4239, BCX-4208); b) la inhibición de enzimas como la PDE4 (AN2728) y la PNP (BCX-4208); c) el bloqueo de los receptores TLR (IMO-8400); d) la inhibición del receptor de la TrkA (CT327); e) la activación de la SIRT1 (SRT2104) y del A3AR (CF101). 2-DG = 2-dexosiguanosina, A3AR = receptor de adenosina $A 3, A M P C=$ monofosfato de adenosina cíclico, D1P = dexosiribosa-1-fosfato, JAK = Janus cinasa, NF-KB, factor nuclear potenciador de las cadenas ligeras kappa de las células $B$ activadas, NGF = factor de crecimiento nervioso, $P N P=$ fosforilasa de nucleósidos de purina, STAT = transductor de señal y activador de la transcripción, $S I R T 1=$ sirtuina- $1, T L R=$ receptores tipo Toll, TrkA = tropomiosina cinasa $A$.

diferenciación terminal de los queratinocitos, está disminuida o ausente en la piel psoriásica, con una capa córnea formada por células no diferenciadas. ${ }^{8}$

En los últimos años se ha puesto en evidencia el papel del sistema inmune en la psoriasis, lo que ha facilitado la comprensión de las bases fisiopatológicas de la enfermedad. Las lesiones psoriásicas se caracterizan por aumento de la infiltración de linfocitos T. Se estima que un paciente con $20 \%$ de la superficie corporal afectada con psoriasis tendrá unos 8 billones de células $T$ en la sangre, mientras que 20 millones se localizarán en la dermis y la epidermis. ${ }^{7}$ Los estudios inmunohistoquímicos confirman que la dermis alberga predominantemente linfocitos T CD4+, macrófagos y células dendríticas (CD), mientras que los linfocitos T CD8 predominan en la epidermis. ${ }^{7}$ A pesar de la indudable importancia del sistema inmunitario en la psoriasis, el debate sobre su causa primaria continúa y numerosas dudas deben resolverse, en particular respecto a la identificación 
de antígenos (exógenos o endógenos) y su interacción con las células epidérmicas e inmunológicas en el contexto de las lesiones cutáneas. ${ }^{9}$

Por estudios genéticos se han identificado nueve locus de susceptibilidad para la psoriasis (PSORS1 a PSORS9). Uno de los principales determinantes genéticos es PSORS1, una región de 220 kb en el complejo mayor de histocompatibilidad localizado en el cromosoma 6p21, con el cual se explica de 35 a $50 \%$ de heredabilidad de la enfermedad. ${ }^{10}$ Otros genes específicos también han sido identificados en distintas poblaciones; sus polimorfismos se han asociado con numerosos procesos inmunológicos, incluyendo la diferenciación de queratinocitos, la proliferación de células T, la modulación de vías de señalización, así como la regulación y adhesión de leucocitos por células T cooperadoras (Th) 1 y $17 . .^{11}$ En fechas recientes también se ha destacado la participación de divide the word as: micro-RNA en la regulación inflamatoria de la enfermedad. ${ }^{12}$

\section{De la investigación básica y clínica al desarrollo de tratamientos antipsoriásicos}

El desarrollo de nuevos tratamientos desde una visión convencional involucra las siguientes etapas:

- Establecimiento de hipótesis etiopatogénicas de la enfermedad.

- Identificación de objetivos farmacológicos.

- Selección de candidatos terapéuticos.

- Optimización química de los compuestos seleccionados.

- Pruebas preclínicas en animales de experimentación.

- Evaluación de la seguridad en seres humanos y establecimiento de las propiedades farmacocinéticas (fase I).

- Pruebas de eficacia (prueba de concepto, fase II).

- Ensayos clínicos multicéntricos a mayor escala para evaluar la seguridad y eficacia (fase III).

En este esquema clásico, el traslado de conocimientos se realiza desde la investigación básica hacia la clínica en un sentido unidireccional.

El desarrollo de medicamentos antipsoriásicos a lo largo de su historia rompe con ese modelo, ya que el traslado de conocimientos no siempre ha partido desde la ciencia básica, sino que muchas hipótesis han surgido a partir de la experiencia clínica pudiendo ser verificadas en investigación fundamental para luego trasladarse nuevamente hacia escenarios clínicos. Así, aunque el concepto de medicina traslacional es relativamente reciente, su implementación en el desarrollo de tratamientos antipsoriásicos no es novedosa.

De hecho, uno de los primeros cambios en cuanto a los paradigmas sobre la patogénesis de la enfermedad se produjo cuando una observación accidental demostró que pacientes trasplantados que recibían ciclosporina evidenciaban mejoría de su psoriasis. ${ }^{13}$ Esta experiencia clínica propició el inicio de nuevas investigaciones que demostraron que la acción de este medicamento reducía el número de linfocitos $T$, células presentadoras de antígenos, monocitos y macrófagos. ${ }^{14}$ En consecuencia, los linfocitos T se consideraron blancos terapéuticos, lo cual propició el desarrollo de una proteína de fusión que selectivamente bloqueaba su activación sin afectar a los queratinocitos. ${ }^{15}$ Curiosamente se utilizaron estrategias de la medicina traslacional que conllevaron a la generación de nuevas hipótesis a partir de las experiencias clínicas (bedside-to-bench).

La hipótesis que consideraba la activación de linfocitos T como un evento central en la etiología de la enfermedad fue posteriormente confirmada con la realización de experimentos en los que el traslado de conocimientos se realizó bidireccionalmente (bench-to-bedside/bedside-to-bench). En uno de ellos se utilizaron ratones inmunosuprimidos en los que se injertó piel no lesionada proveniente de pacientes con psoriasis. Esta piel adquirió características de placas psoriásicas después de la inyección de leucocitos. ${ }^{16}$ Para probar la misma hipótesis se usaron antagonistas específicos (anticuerpos antiCD80 y proteína de fusión CTLA4-Ig) de moléculas de superficie implicadas en la activación de los linfocitos T. ${ }^{17}$ Estos fueron experimentos clínicos acoplados con investigaciones básicas que condujeron a la elucidación de vías de señalización vinculadas con la patogénesis de la enfermedad.

En esta época se extendió la idea de que la presencia de una red de citocinas generadas por los linfocitos en individuos genéticamente predispuestos tenía influencia en la hiperproliferación de los queratinocitos. Como consecuencia se diseñaron productos biotecnológicos para bloquear la acción de dichas citocinas. Un ejemplo de ello fue el daclizumab, un anticuerpo monoclonal dirigido contra el receptor de la IL-2 y la salida al mercado de dos nuevos agentes dirigidos a inactivar a los linfocitos T: alefacept y efalizumab. Aunque efalizumab fue retirado más tarde por generar efectos adversos graves, su uso constituyó otra prueba de la importancia de los linfocitos T en esta patología. 
En la década de 1990 ya se había demostrado que el factor de necrosis tumoral (TNF) se encontraba en altas concentraciones en las placas psoriásicas. ${ }^{18} \mathrm{La}$ remisión de las lesiones cutáneas en pacientes con artritis psoriásica luego del uso de un agente anti-TNF (etanercept) ${ }^{19}$ puso en evidencia el papel relevante de esta citocina y de la respuesta inmunológica innata en la psoriasis. ${ }^{20}$ Estudios clínicos posteriores, complementados con herramientas de biología celular y molecular, confirmaron que los agentes anti-TNF también tenían efectos sobre la proliferación de otros linfocitos, los Th17. ${ }^{21}$ Estos abordajes bidireccionales de medicina traslacional contribuyeron a la identificación de mediadores clave en la enfermedad, permitiendo la salida al mercado de fármacos con mayor selectividad sobre citocinas específicas.

Aunque los primeros medicamentos anti-TNF surgieron a principios del siglo XXI (etanercept, adalimumab, infliximab), nuevas estrategias farmacológicas han permitido el desarrollo de otros agentes anti-TNF novedosos. Es el caso del certolizumab pegol, que difiere de otros fármacos anti-TNF por su estructura. Este medicamento está compuesto por un fragmento Fab de un anticuerpo monoclonal murino humanizado anti-TNF combinado con dos moléculas de polietilenglicol, 22 lo que incrementa su tiempo de vida media a aproximadamente 14 días con mayor distribución tisular. ${ }^{22} \mathrm{Al}$ estar libre de la porción Fc, el certolizumab pegol no forma complejos inmunes ni induce citotoxicidad mediada por anticuerpos ni por complemento. Además, posee una afinidad por el TNF superior a otros medicamentos como infliximab y adalimumab, ${ }^{22} \sin$ inducir apoptosis en linfocitos $T$ y monocitos de individuos sanos.

La importancia de las células Th17 en la psoriasis fue también establecida utilizando enfoques de medicina traslacional. Primeramente, la existencia de este grupo de células productoras de IL-17, su impacto en enfermedades inflamatorias autoinmunes y su importante presencia en piel y sangre periférica de individuos con psoriasis se demostró en modelos animales y pacientes. ${ }^{23,24}$ Otras investigaciones evidenciaron que la activación de las células Th17 estaba influida por la IL-23 $23^{25}$ y que esta compartía la subunidad p40 con la IL-12. ${ }^{26}$ Estos estudios de ciencia básica combinados con comprobaciones clínicas permitieron el establecimiento de un nuevo paradigma en la fisiopatología de la enfermedad relacionado con el eje IL-23/ IL-17. ${ }^{27}$ La consecuencia lógica de estas investigaciones fue el desarrollo de dos anticuerpos contra la subunidad p40: el ustekinumab y briakinumab, así como de inhibidores de la IL-17 o de su receptor (secukinumab, ixekizumab, brodalumab).

Un análisis exhaustivo de lo sucedido hace dos décadas con la generación de nuevos medicamentos para la psoriasis permite comprender el impacto de la medicina traslacional en este proceso. De hecho, el éxito de los biotecnológicos se debió a la realización de investigaciones en las que se establecieron hipótesis en modelos biológicos con posibilidad de trasladarse rápidamente al campo clínico, al mismo tiempo que esta experiencia clínica servía de base para refinar los conceptos etiopatogénicos.

Este proceso bidireccional ha garantizado el éxito en la generación de tratamientos $y$, aunque muchos de ellos han quedado en el camino (anticuerpo anti-IL8, anticuerpo anti-CD5, anticuerpo anti-L selectina), no cabe duda de que esta estrategia ha permitido la salida al mercado de nuevos fármacos, contribuyendo a la mejoría en la calidad de vida de los pacientes.

\section{Nuevos fármacos antipsoriásicos en desarrollo}

La comprensión más profunda de los eventos celulares y moleculares implicados en la etiología de la psoriasis ha permitido que se haya producido un salto considerable en cuanto al número de fármacos en desarrollo asociados con nuevos objetivos terapéuticos (Figura 1). Destacan los que activan o bloquean la fosfodiesterasa 4, el receptor de la tropomiosina cinasa A, la fosforilasa de nucléosidos de purina, el receptor de adenosina $A 3$, el receptor de esfingosina-1-fosfato y la familia de las Janus cinasas, entre otros (Tabla 1).

\section{Perspectivas futuras en el desarrollo de nuevos medicamentos antipsoriásicos}

El uso de estrategias de medicina traslacional en el desarrollo de medicamentos antipsoriásicos ha permitido en los últimos 20 años incrementar significativamente el número de los disponibles para esta patología. La salida al mercado de los productos biotecnológicos constituyó un hito en los esquemas de desarrollo de este tipo de fármacos, ya que combinó conscientemente una estrategia bidireccional donde los conocimientos derivados de la ciencia básica se incorporaron rápidamente a la investigación clínica, al mismo tiempo que la experiencia clínica sirvió de base para aceptar o refutar hipótesis de la ciencia básica. Por ello, no resulta sorprendente que existan 18 
Tabla 1. Nuevos medicamentos en desarrollo para el tratamiento de la psoriasis

\begin{tabular}{|c|c|c|}
\hline Fármaco & Mecanismo de acción/Objetivo terapéutico (Compañía farmacéutica) & Fase del desarrollo \\
\hline AN2728 & Inhibidor selectivo de la fosfodiesterasa 4 (Pfizer) & Fase $\|^{28}$ \\
\hline СТ327 & Inhibidor selectivo del receptor de la tropomiosina cinasa A (TrkA) (Creabilis, S.A) & Fase $\|^{29}$ \\
\hline IMO-8400 & $\begin{array}{l}\text { Bloqueo de la activación de los receptores de tipo Toll (TLR) 7,8 y } 9 \text { (Idera } \\
\text { Pharmaceuticals) }\end{array}$ & Fase $\|^{30}$ \\
\hline SRT2104 & $\begin{array}{l}\text { Activa a la sirtuina-1 (SIRT1) modulando la función de NF-кB y p53, inhibe la } \\
\text { proliferación de queratinocitos (GlaxoSmithKline) }\end{array}$ & Fase $\|^{31}$ \\
\hline AZD0284 & $\begin{array}{l}\text { Agonista inverso del receptor de ácido retinoide asociado con el receptor nuclear } \\
\text { huérfano (AztraZeneca) }\end{array}$ & Fase $\|^{32}$ \\
\hline CF101 & Agonista del receptor de adenosina A3 (A3AR) (Can-Fite BioPharma Ltd) & Fase $\|^{33}$ \\
\hline BCX-4208 & $\begin{array}{l}\text { Inhibidor de la fosforilasa de nucleósidos de purina (PNP) (BioCryst Pharmaceuticals, } \\
\text { Inc/Hoffman-La Roche) }\end{array}$ & Fase $\|^{34}$ \\
\hline $\begin{array}{l}\text { Namilumab } \\
\text { (MT-203) }\end{array}$ & $\begin{array}{l}\text { Anticuerpo monoclonal que neutraliza de forma potente y específica al factor estimulante } \\
\text { de colonias de granulocitos y macrófagos (GM-CSF) (Takeda Pharmaceutical) }\end{array}$ & Fase $\|^{35}$ \\
\hline $\begin{array}{l}\text { Ponesimod } \\
\text { (ACT-128800) }\end{array}$ & $\begin{array}{l}\text { Agonista selectivo del receptor de esfingosina-1-fosfato (S1P) reduce la disponibilidad } \\
\text { de células efectoras B/T circulantes. (Actelion Pharmaceuticals) }\end{array}$ & Fase $\|^{36}$ \\
\hline RWJ-445380 & Inhibidor de la catepsina S (Alza Corporation, DE) & Fase $\|^{37}$ \\
\hline Ruxolitinib & Inhibidor de la familia de las cinasas Janus JAK1/JAK2 (Incyte Corporation) & Fase $\|^{38}$ \\
\hline Baricitinib & Inhibidor de la familia de las cinasas Janus JAK1/JAK2 (Eli Lilly) & Fase $\|^{39}$ \\
\hline GSK2586184 & Bloqueador selectivo de JAK1 (GlaxoSmithKline) & Fase $\|^{40}$ \\
\hline INCB039110 & Bloqueador selectivo de JAK1 (Incyte Corporation) & Fase $\|^{41}$ \\
\hline Lestaurtinib & Inhibidor del receptor de tirosina cinasa FLT3 y de JAK2 (Teva Pharmaceutical) & Fase $\|^{42}$ \\
\hline ASP015K & Inhibidor selectivo de JAK3 (Astellas Pharma) & Fase $\|^{43}$ \\
\hline MOL4239 & $\begin{array}{l}\text { Inhibidor de la fosforilación del transductor de señal y activador de la transcripción } 3 \\
\text { (STAT3) (Moleculin, LLC) }\end{array}$ & Fase $I^{44}$ \\
\hline KD025 & $\begin{array}{l}\text { Inhibidor de la quinasa } 2 \text { asociada con Rho (ROCK2) bloqueando la fosforilación de } \\
\text { STAT3 y la producción de IL-17 e IL-21(Kadmon Holdings, Inc) }\end{array}$ & Fase $\|^{45}$ \\
\hline
\end{tabular}

fármacos antipsoriásicos asociados con nuevos objetivos, los cuales que están siendo desarrollados por múltiples compañías farmacéuticas en el mundo.

A pesar de estas alentadoras noticias, la psoriasis sigue siendo una enfermedad incurable y existen todavía varios aspectos que pueden dificultar el desarrollo futuro de fármacos, que deben ser profundizados en su integración con esquemas de medicina traslacional. Como ejemplo podrían enumerarse los siguientes:

- La caracterización de antígenos específicos responsables de la activación de la respuesta inmunológica.

- La identificación de biomarcadores predictivos de toxicidad, farmacocinética y eficacia farmacológica.

- El desarrollo de modelos in vivo e in vitro más representativos de la enfermedad.
- La integración de aspectos genómicos al proceso del descubrimiento de fármacos.

Mucho queda por comprender las causas de esta enfermedad, así como la influencia de marcadores genéticos en la respuesta a nuevos tratamientos, pero seguramente el traslado bidireccional de conocimientos permitirá la introducción futura de nuevas opciones individualizadas en las que la cooperación entre todos los actores (científicos, médicos, pacientes) será necesaria para el cambio de paradigmas.

\section{Bibliografía}

1. Parisi R, Symmons DPM, Griffiths CEM, Ashcroft DM. Global epidemiology of psoriasis: a systematic review of incidence and prevalence. $\mathrm{J}$ Invest Dermatol. 2013;133:377-385.

2. International Psoriasis Council. [Sitio web]. IPC psoriasis review. Dallas, Texas: IPC; 2009. p. 1-12. [Consultado 2017 Sep 10]. Disponible en: http://www.psoriasiscouncil.org/docs/ipcpsoriasisreview_dec_2009_ english.pdf?LanguageID=EN-US 
3. Kurd SK1, Troxel AB, Crits-Christoph P, Gelfand JM. The risk of depression, anxiety, and suicidality in patients with psoriasis: a population-based cohort study. Arch Dermatol. 2010;146:891-5.

4. Nast A, Boehncke WH, Mrowietz U, Ockenfels HM, Philipp S, Reich K, et al. S3-Guidelines on the treatment of psoriasis vulgaris (English version). Update. J Dtsch Dermatol Ges. 2012;10:S1-S95

5. Dubertret L, Mrowietz U, Ranki A, Van-De-Kerkhof PC, Chimenti S Lotti T, et al. European patient perspectives on the impact of psoriasis: the EUROPSO patient membership survey. $\mathrm{Br} J$ Dermatol. 2006; 155:729-736

6. Lowe NJ, Wieder JM, Rosenbach A, Johnson K, Kunkel R, Bainbridge C et al. Long-term low-dose cyclosporine therapy for severe psoriasis: effects on renal function and structure. J Am Acad Dermatol. 1996; 35:710-719.

7. Cai Y, Fleming C, Yan J. New insights of T cells in the pathogenesis of psoriasis. Cell Mol Immunol. 2012;9:302-309.

8. Lowes MA, Bowcock AM, Krueger JG. Pathogenesis and therapy of psoriasis. Nature. 2007;445:866-873.

9. Guttman-Yassky E, Krueger JG. Psoriasis: evolution of pathogenic concepts and new therapies through phases of translational research. $\mathrm{Br} \mathrm{J}$ Dermatol. 2007;157:1103-1115.

10. Hébert HL, Ali FR, Bowes J, Griffiths CE, Barton A, Warren RB. Genetic susceptibility to psoriasis and psoriatic arthritis: implications for therapy. Br J Dermatol. 2012;166:474-482.

11. Das S, Stuart PE, Ding J, Tejasvi T, Li Y, Tsoi LC, et al. Fine mapping of eight psoriasis susceptibility loci. Eur J Hum Genet. 2015;23:844-853.

12. Hawkes JE, Nguyen GH, Fujita M, Florell SR, Callis Duffin K, Krueger GG, et al. microRNAs in psoriasis. J Invest Dermatol. 2016;136:365-371.

13. Picascia DD, Garden JM, Freinkel RK, Roenigk HH. Resistant severe psoriasis controlled with systemic cyclosporine therapy. Transplant Proc. 1988;20:58-62.

14. Griffiths CE, Voorhees JJ. Cyclosporine A in the treatment of psoriasis: a clinical and mechanistic perspective. J Invest Dermatol. 1990;95:S53-S55

15. Gottlieb SL, Gilleaudeau P, Johnson R, Estes L, Woodworth TG, Gottlieb $A B$, et al. Response of psoriasis to a lymphocyte-selective toxin (DAB389IL-2) suggests a primary immune, but not keratinocyte, pathogenic basis. Nat Med. 1995;1:442-447

16. Wrone-Smith T, Nickoloff BJ. Dermal injection of immunocytes induces psoriasis. J Clin Invest. 1996;98:1878-1887.

17. Gottlieb AB, Lebwohl M, Totoritis MC, Abdulghani AA, Shuey SR, Romano $P$, et al. Clinical and histologic response to single-dose treatment of moderate to severe psoriasis with an anti-CD80 monoclonal antibody. J Am Acad Dermatol. 2002;47:692-700.

18. Nickoloff BJ, Karabin GD, Barker JN, Griffiths CEM, Sarma V, Mitra RS et al. Cellular localization of interleukin-8 and its inducer, tumor necrosis factor-alpha in psoriasis. Am J Pathol. 1991:138:129-140.

19. Mease PJ, Goffe BS, Metz J, VanderStoep A, Finck B, Burge DJ. Etanercept in the treatment of psoriatic arthritis and psoriasis: a randomised trial. Lancet. 2000;356:385-390.

20. Victor FC, Gottlieb AB. TNF-alpha and apoptosis: implications for the pathogenesis and treatment of psoriasis. J Drugs Dermatol. 2002 1:264-275

21. Zaba LC, Cardinale I, Gilleaudeau $P$, Sullivan-Whalen $M$, Suárez-Fariñas M, Fuentes-Duculan J, et al. Amelioration of epiderma hyperplasia by TNF inhibition is associated with reduced Th17 responses. J Exp Med. 2007:204:3183-3194.

22. Pasut G. Pegylation of biological molecules and potential benefits: pharmacological properties of certolizumab pegol. BioDrugs. 2014;28:S15-S23.

23. Harrington LE, Hatton RD, Mangan PR, Turner H, Murphy TL, Murphy KM, et al. Interleukin 17-producing CD4+ effector T cells develop via a lineage distinct from the T helper type 1 and 2 lineages. Nat Immunol. 2005;6:1123-1132.

24. Lowes MA, Kikuchi T, Fuentes-Duculan J, Cardinale I, Zaba LC, Haider AS, et al. Psoriasis vulgaris lesions contain discrete populations of Th1 and Th17 T cells. J Invest Dermatol. 2008;128:1207-1211.

25. McKenzie BS, Kastelein RA, Cua DJ. Understanding the IL-23-IL-17 immune pathway. Trends Immunol. 2006;27:17-23.

26. Oppmann B, Lesley R, Blom B, Timans JC, Xu Y, Hunte B, et al. Nove p19 protein engages IL-12p40 to form a cytokine, IL-23, with biologica activities similar as well as distinct from IL-12. Immunity. 2000; 13:715-725.

27. Di-Cesare A, Di-Meglio P, Nestle FO. The IL-23/Th17 axis in the immunopathogenesis of psoriasis. J Invest Dermatol. 2009;129:1339-1350.
28. Nazarian R, Weinberg JM. AN-2728, a PDE4 inhibitor for the potential topical treatment of psoriasis and atopic dermatitis. Curr Opin Investig Drugs. 2009;10:1236-1242

29. Roblin D, Yosipovitch G, Boyce B, Robinson J, Sandy J, Mainero V, et al. Topical TrkA kinase inhibitor CT327 is an effective, novel therapy for the treatment of pruritus due to psoriasis: results from experimental studies, and efficacy and safety of CT327 in a phase $2 b$ clinical trial in patients with psoriasis. Acta Derm Venereol. 2015; 95:542-548.

30. Balak DMW, Van-Doorn MBA, Rissmann R, Sullivan T, Burggraaf J, Arbeit RD. Results from a randomized, double-blind, placebo-controlled, monotherapy trial of IMO-8400 demonstrate clinical proof-of-concept for Toll-like receptor 7, 8, and 9 antagonism in psoriasis. J Am Acad Dermatol. 2015;72:AB247.

31. Krueger JG, Suárez-Fariñas M, Cueto I, Khacherian A, Matheson R, Parish LC, et al. A randomized, placebo-controlled study of SRT2104, a SIRT1 activator, in patients with moderate to severe psoriasis. PLoS ONE. 2015;10:e0142081.

32. ClinicalTrials.gov. [Sitio web]. Study to assess the safety, tolerability and pharmacokinetics and pharmacodynamics of AZD0284 in healthy subjects. [Consultado 2017 Sep 10]. Disponible en: https://clinicaltrials.gov/ ct2/show/NCT02976831?term $=A Z D 0284 \&$ rank $=2$

33. David M, Gospodinov DK, Gheorghe N, Mateev GS, Rusinova MV, Hristakieva E, et al. Treatment of plaque-type psoriasis with oral CF101: data from a phase II/III multicenter, randomized, controlled trial. J Drugs Dermatol. 2016;15:931-938.

34. Bantia S, Parker C, Upshaw R, Cunningham A, Kotian P, Kilpatrick JM, et al. Potent orally bioavailable purine nucleoside phosphorylase inhibitor BCX-4208 induces apoptosis in B- and T-lymphocytes. A novel treatment approach for autoimmune diseases, organ transplantation and hematologic malignancies. Int Immunopharmacol. 2010;10:784-790.

35. ClinicalTrials.gov. [Sitio web]. Efficacy and safety of namilumab (MT203) for plaque psoriasis. [Consultao 2017 Sep 10]. Disponible en: https:// clinicaltrials.gov/ct2/show/NCT02129777?term=Namilumab\&rank=1

36. Vaclavkova A, Chimenti S, Arenberger P, Holló P, Sator PG, Burcklen M, et al. Oral ponesimod in patients with chronic plaque psoriasis: a randomized, double-blind, placebo-controlled phase 2 trial. Lancet. 2014; 384:2036-2045.

37. ClinicalTrials.gov. [Sitio web]. Study to investigate the safety, tolerability, absorption, distribution, metabolism, and elimination of RWJ-445380 administered to patients with plaque psoriasis. [Consultado 2017 Sep 10]. Disponible en: https://clinicaltrials.gov/ct2/show/NCT00396422?ter$\mathrm{m}=\mathrm{RWJ}-445380$ \&rank $=1$

38. Punwani N, Scherle P, Flores R, Shi J, Liang J, Yeleswaram S, et al. Preliminary clinical activity of a topical JAK1/2 inhibitor in the treatment of psoriasis. J Am Acad Dermatol. 2012;67:658-664

39. Papp KA, Menter MA, Raman M, Disch D, Schlichting DE, Gaich C, et al. A randomized phase $2 b$ trial of baricitinib, an oral Janus kinase (JAK) 1/ JAK2 inhibitor, in patients with moderate-to-severe psoriasis. Br J Dermatol. 2016:174:1266-1276.

40. Ludbrook VJ, Hicks KJ, Hanrott KE, Patel JS, Binks MH, Wyres MR, et al. Investigation of selective JAK1 inhibitor GSK2586184 for the treatment of psoriasis in a randomized placebo-controlled phase lla study. $\mathrm{Br}$ J Dermatol. 2016;174:985-995.

41. Bissonnette R, Luchi M, Fidelus-Gort R, Jackson S, Zhang H, Flores R, et al. A randomized, double-blind, placebo-controlled, dose-escalation study of the safety and efficacy of INCB039110, an oral janus kinase 1 inhibitor, in patients with stable, chronic plaque psoriasis. J Dermatol Treat. 2016;27:332-338.

42. ClinicalTrials.gov. [Sitio web]. Study of the efficacy, safety and tolerability of oral CEP-701 in patients with severe psoriasis. [Consultado 2017 Sep 10]. Disponible en: https://clinicaltrials.gov/ct2/show/NCT00236119?term =Lestaurtinib\&cond=psoriasis\&rank $=1$

43. Papp KA, Pariser D, Catlin M, Wierz G, Ball G, Akinlade B, et al. A phase 2 a randomized, double-blind, placebo-controlled, sequential dose-escalation study to evaluate the efficacy and safety of ASP015K, a novel Janus kinase inhibitor, in patients with moderate-to-severe psoriasis. $\mathrm{Br}$ J Dermatol. 2015;173:767-776.

44. ClinicalTrials.gov. [Sitio web]. Paired psoriasis lesion, comparative, study to evaluate MOL4239 in psoriasis. [Consultado 2017 Sep 10]. Disponible en: https://clinicaltrials.gov/ct2/show/NCT01826201?term=MOL4239\&rank=1

45. Zanin-Zhorov A, Weiss JM, Nyuydzefe MS, Chen W, Scher JU, Mo R et al. Selective oral ROCK2 inhibitor down-regulates IL-21 and IL-17 secretion in human T cells via STAT3-dependent mechanism. Proc Natl Acad Sci U S A. 2014;111:16814-16819. 